\title{
Research on Mathematical Dialectical Logic for Intelligent Information Processing ${ }^{\dagger}$
}

\author{
Huacan He ${ }^{1, *}$, Yanquan Zhou ${ }^{2}$ and Zhicheng Chen ${ }^{3}$ \\ 1 School of Computer, Northwestern Polytechnical University, Xi'an 710072, China \\ 2 School of Computer, Beijing University of Posts and Telecommunications, Beijing 100876, China; \\ zhouyanquan@bupt.edu.cn \\ 3 Room 208 of Building A, Zhongguancun Intelligent Manufacturing Way, Haidian District, Beijing 100083, \\ China; gfdtek@163.com \\ * Correspondence: hehuac@nwpu.edu.cn; Tel.: +86-136-9130-9054 \\ † Presented at the IS4SI 2017 Summit DIGITALISATION FOR A SUSTAINABLE SOCIETY, Gothenburg, \\ Sweden, 12-16 June 2017.
}

Published: 9 June 2017

\begin{abstract}
Information ecology requires the support of intelligent information processing, while the latter requires the support of mathematical dialectical logic. This paper introduces the research status and prospect of mathematical dialectical logic for intelligent information processing, including: several basic assumptions (axioms) about information and intelligence; based on mathematical formal logic, gradually liberalizing the constraints to establish the research compendium of mathematical dialectical logic theory system; according to the forming mechanism of various uncertainties, the principles and methods of defining and generating the complete operator cluster of mathematical dialectical logic on propositional level, establishing the complete operator library of intelligent information processing; two application methods of the operator library in intelligent information processing; future work.
\end{abstract}

Keywords: information ecology; intelligent information processing; mathematical dialectical logic; uncertainty reasoning; operator library

\section{The Basic Assumptions of Information and Intelligence}

Our research based on the fundamental beliefs from the writer, which can be used widely as well. They are listed below:

A1. The universe consists of two words and four elements.

The universe is an antithetic and synthetic party composed of the material world and the information world.

The material object has the space-occupancy (Performance is the exclusivity of the object) and inertia (Performance as the mobile object needs to pay force and energy) in the material world. Matter structure, mass, force and energy are fundamental problems in the material world.

The information object has the space-occupancy (Performance is the exclusivity of the object) and inertia (Performance as the mobile object needs to pay intelligence and intelligent) in the possibility space. Information structure, amount of information, intelligence and intelligent are the basic problems in the information world.

We believe in the following scientific hypothesis: The universe is made up of numerous informofer with duality. The opening and closing status of the duality decides whether it is in information-state or material-state. So the universe between the two worlds can be linked and converted. Time may be the synchronizing signal of two worlds. 
A2. Uncertainty is the essential feature of the universe.

There exists a transformation from determinism to evolution. In the era of energy, people were facing closed simple mechanical system, which leads to determinism: they tended to believe that the development was controlled by unchangeably objective law and time is a scalar. Uncertainty is caused by the incapability of catching all of the objective law and state parameters of the research object. The method to solve it is reductionism, of which the fundamental theories are calculus and Standard logic.

In the era of information, the systems we facing are opening complex evolution system, where everything is constantly changing. New regular pattern arise in the meantime of new things appearing. This lead to a new theory: the universe is inherently uncertain, for it is evolving continuously. Consequently, time may be a vector. This is the theory of evolution. Due largely to the limitation that people can only know part of the information from our world, we can't get rid of the uncertainty absolutely. As a result, it is required that we should consider the elements about dialectical contradiction, emergence effect, ecological balance, etc. The fundamental needs spatial analysis, Universal Logics and multi-objective coordination.

\section{A3. Distinguishing the two different kinds of contradiction is strictly necessary.}

Standard logic get rid of any contradiction. On the contrary, Universal Logics strictly distinguish logical contradiction and dialectical contradiction, for they are two kinds of different contradiction. A logical contradiction is a deterministic error and it is not allowed in any logical system, so it must be excluded. For example, a proposition A is determined to be true and false at the same time in the binary logic, the truth degree of a proposition B is 0.7 and 0.4 at the same time in the continuous value logic. These logical contradictions are not allowed. Dialectical contradiction is the object of universal logic research. It is ubiquitous and must be researched positively. For example, determining the coin with positive and negative side at the same time is an antithetic and synthetic party in the binary logic. This dialectical contradiction cannot be ruled out. If the proposition A (the coin is positive) is judged to be true and false at the same time, this is a logical contradiction that must be excluded. For another example, in the continuous value logic, the truth degree of Proposition B is 0.7 , it means that the false degree of B has been judged to be 0.3 . The situation that true and false exist in the proposition truth is a dialectical contradiction, it cannot be excluded, and otherwise the continuous value logic is degraded to binary logic.

\section{The Research Program of Universal Logics}

\subsection{Origin}

In the early years, the establishment and development of the artificial intelligence school are based on information processing, and its logical paradigm is the full implementation of the "not this but that" standard logic. Both the Turing machine model and the perception model demonstrate that the standard logic is a Universal Logics which describes all the information processing processes that meet the "not this but that" nature of the world.

The crisis of artificial intelligence in the 1980s has exposed the limitation of standard logic. It is very inefficient in practical application that cannot overcome the combination explosion of information processing algorithms due to the high complexity of space and time .It was helpless and at a loss when facing a variety of objective existence of uncertainty (known as dialectical contradictions).

Dozens of non-standard logic that devoted to certain uncertainties (dialectical contradictions) have sprung up as an emergency response to the theoretical crisis, which can be more effective to solve some practical application problems. But sometimes there would be some inexplicable abnormal results so people can not accurately grasp the effective use of these non-standard logic to eliminate the emergence of abnormal results. 
Can we make an abstraction, similar as Mendeleev's periodic table, for the framework of Universal Logics which is a multi-dimensional space with the standard logic as original point, the indeterminacy as coordinate axis? In such framework, each possibility of non-standard logics will be one point of the space in continuous distribution. Based on the kinds of indeterminacy for the handled information, users can choose the related logic points in the universal logics.

The following sections detail the steps and objectives of the extended standard logic for the universal logic framework.

\subsection{Basic Principle}

A core issue: In the absence of a logical contradiction, tolerance of various dialectic contradictions (uncertainty and evolution).

Two basic routes: Determined a proposition by projecting it to a subspace through space-time positioning; introducing the flexible parameters and the corresponding adjustment mechanism to adjust the logic operator.

Three breakthrough directions: The number of proposition truth values, the completeness of reasoning information and the dimension of the true value space.

Four logic elements: Domain of definition, propositional connective, quantifier and reasoning scheme (See Figure 1).

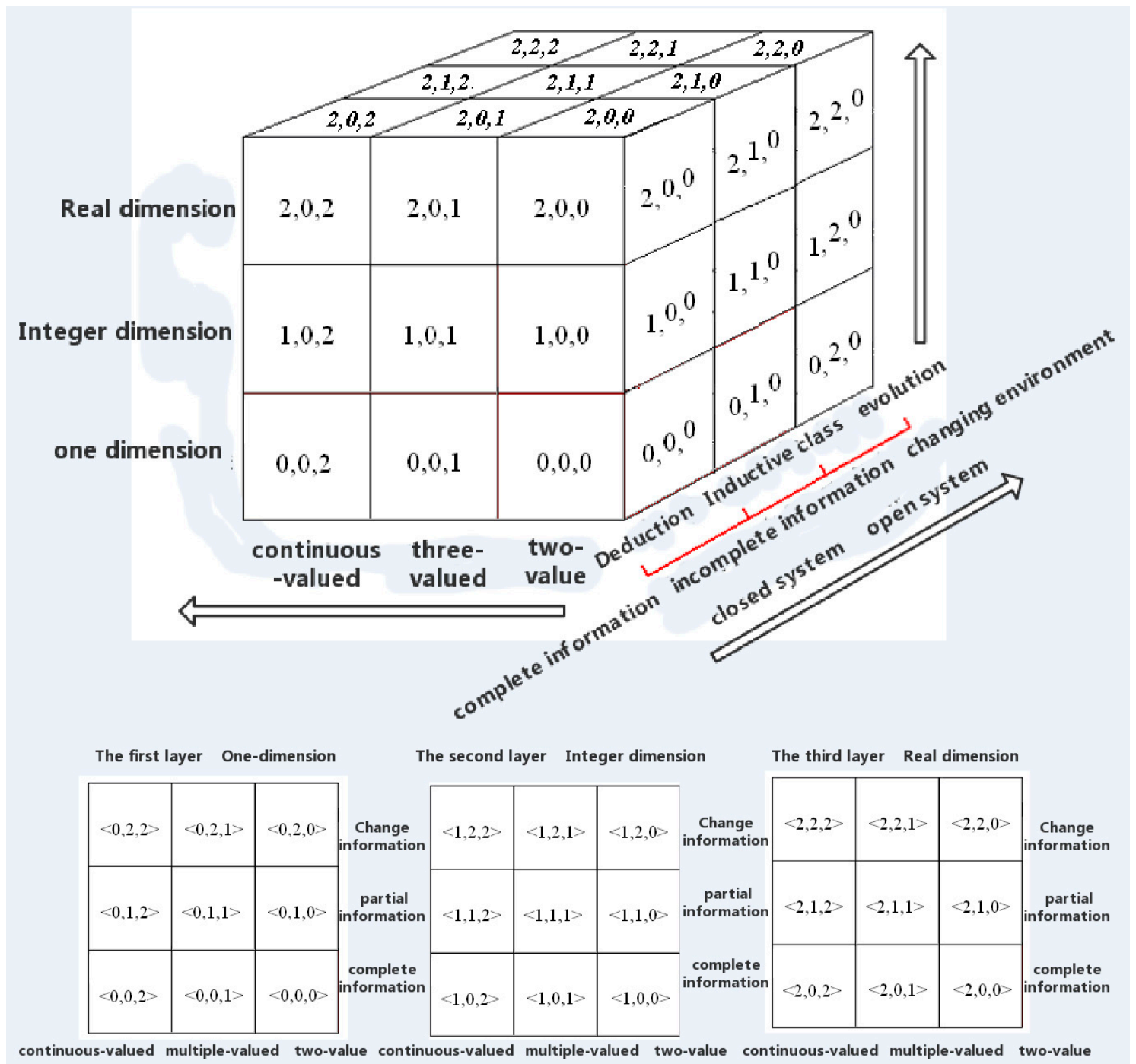

Figure 1. Expand the standard logic $(<0,0,0>$ area $)$ into three directions of the Universal Logics. 
2.3. The Three Domains of the Universal Logics $W=<\{\perp\} \cup$ Range $<\alpha>$, Domain, Model Domain $>$

Table 1. The three domains of the Universal Logics.

\begin{tabular}{ccc}
\hline Range & Domain & Model Domain \\
\hline$[0,1]^{n}, n>0$ & Multigranularity & Continuous state \\
{$[0,1]^{n}, n=1,2,3, \ldots$} & Singlegranularity & Multistate \\
{$[0,1]$ Continuous } & & Singlestate \\
$\{0, \mathrm{u}, 1\}$ Triple & & \\
$\{0,1\}$ Two & & \\
\hline
\end{tabular}

2.4. Layer up to Establish a Variety of Reasoning Model

Table 2. Layer up to establish a variety of reasoning model.

\begin{tabular}{cll}
\hline Feature & \multicolumn{1}{c}{ Inference Mechanism } & \multicolumn{1}{c}{ Inference Model } \\
$\begin{array}{c}\text { Changing } \\
\text { information }\end{array}$ & $\begin{array}{l}\text { Dynamic interaction and balance mechanism of } \\
\text { internal and external parameters }\end{array}$ & Evolutionary reasoning \\
\hline $\begin{array}{c}\text { Incomplete } \\
\text { information }\end{array}$ & $\begin{array}{l}\text { Containment and correction mechanisms for } \\
\text { errors }\end{array}$ & $\begin{array}{l}\text { Sub coordination reasoning; Non monotonic } \\
\text { reasoning; Fault-tolerant reasoning }\end{array}$ \\
\cline { 2 - 3 } & Reasoning based on certain assumptions & $\begin{array}{l}\text { Analogic, hypothetical deduction, case-based } \\
\text { reasoning }\end{array}$ \\
\cline { 2 - 3 } & From special premise to general hypothesis & $\begin{array}{l}\text { Incompletely inductive andDiscovery } \\
\text { reasoning }\end{array}$ \\
\hline $\begin{array}{c}\text { Complete } \\
\text { information }\end{array}$ & From a special premise to a general conclusion & Completely inductive inference \\
\cline { 2 - 3 } Fundamental theory & From general premise to special conclusion & Deductive inference \\
\cline { 2 - 3 } & Propositional connectives, quantifier & Predicate calculus \\
\hline
\end{tabular}

\subsection{Introduce Various Flexible Quantifier to Universal Logics}

Dialectical quantifier defined in $\mathrm{W}$ :

- Threshold element quantifier signing the truth value's deviation in proposition $0^{\text {kt }}$

- Hypothetical quantifier indicating the Degree of trust of a proposition $\$^{k}$

- Scope quantifier restricting Individual variable range $\oint k$

- Position quantifier indicating Relative position of individual variables $q k$

- Transition quantifiers changing the transition properties of truth value distributions $\int^{k}$

In addition, $k \in[0,1]$ is a constraint condition, which is regarded as Degree flexibility, describes the uncertainty.

\subsection{The Application Needs to Gradually Expand as the Establishment of Universal Logic Is a Gradual Growth Process}

The research object of the universal logics is the problem of handling the information with internal identity and external determinacy. As for the constant of the element of logistics, there is only one equivalence system. The universal logics need to deal with the dialectical antinomy. However, each element of logics may bring in different flexibility arguments and modification mechanisms, which evolves infinite un-equivalence systems. Thus, building universal logics is an infinite and incremental development process.

\section{A Variety of Universal Propositional Logic Operator of Integrity Cluster (Library)}

The following describes the principles and methods of introducing various propositional logic-level uncertainties, which can describe and deal with realistic problems in the objective world that contain various propositional-level uncertainties (dialectical contradictions) without causing any logical contradictions. 


\subsection{The Comparative Analysis of Four Continuous Logic Operator}

We can see that the boundary conditions of the four groups of operators are consistent and can be monotonically changed with the input variables from Figure 2 . The fuzzy logic uses the superior limits operator, followed by the probability logic and the continuous logic, while the drastic logic uses the inferior limits operator.

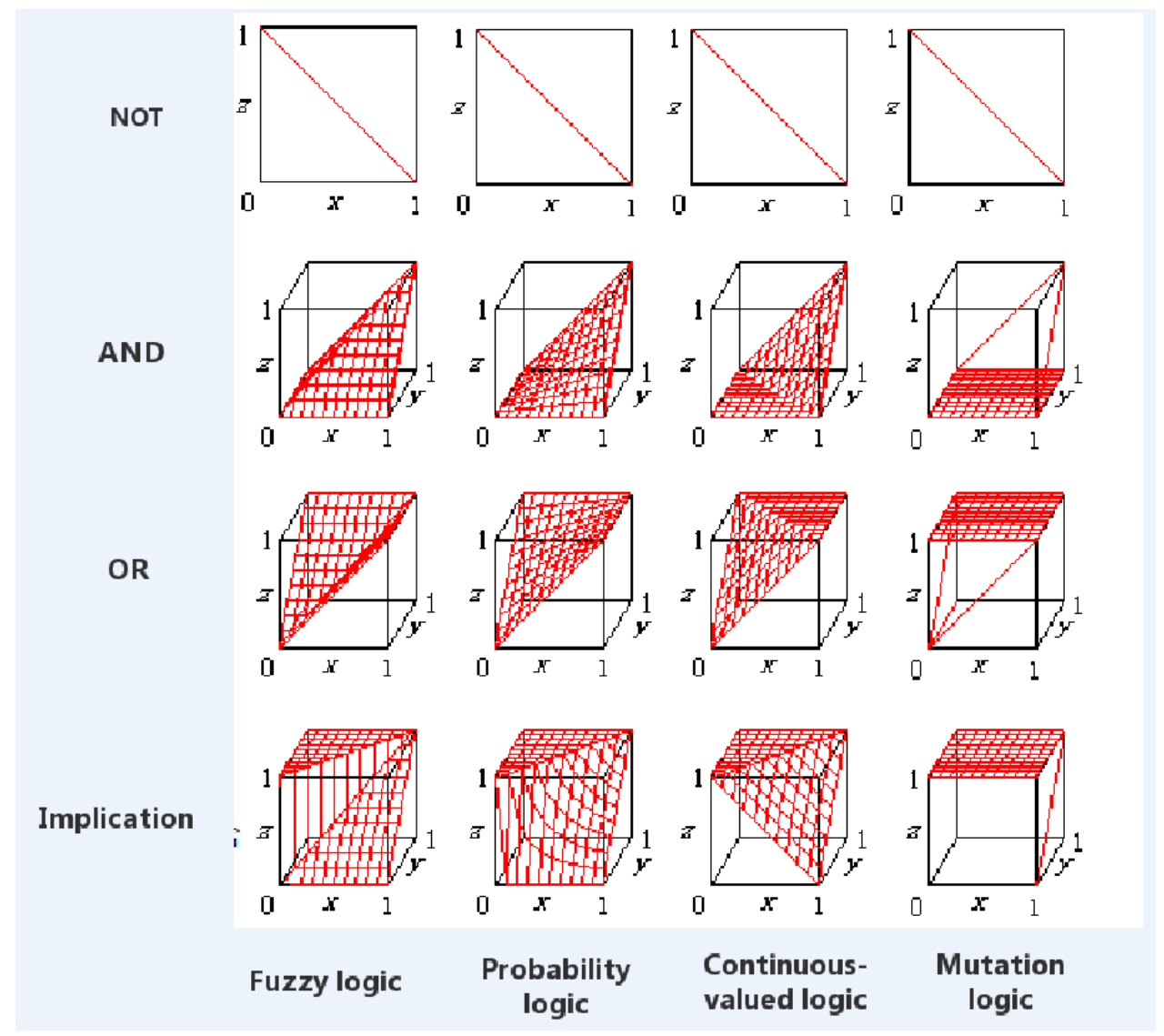

Figure 2. The comparison of four Continuous logic operator.

According to Figure 2 people may ask, whether there are other logical operators exist in the gap between the four logical operators? If so, what logics are they? If these logic are continually arranged and infinitely many, they continuous change from the fuzzy logic to the probability logic, and then to the continuous logic, and then to the end of the drastic logic, is a complete logical operator cluster. Then the flexible change process of thinking can be described by the flexible logical cluster! Of course, to figure out this problem, we must integrate all kinds of non-standard logic results, find a breakthrough from the integration!

\subsection{Find the Breakthrough}

By studying the triangular norm theory, we can found that the four non-standard logic operators are four special points in the Schweizer operator cluster, which are continuously changing (Figure 3). The formal parameter of Schweizer operator cluster $m(-\infty, \infty)$, when $m \rightarrow-\infty$, it is fuzzy logic operator, when $m=0$, it is probability logic operator, when $m=1$, it is a continuous logic operator, and when $\mathrm{m} \rightarrow \infty$, it is drastic logic operator. 

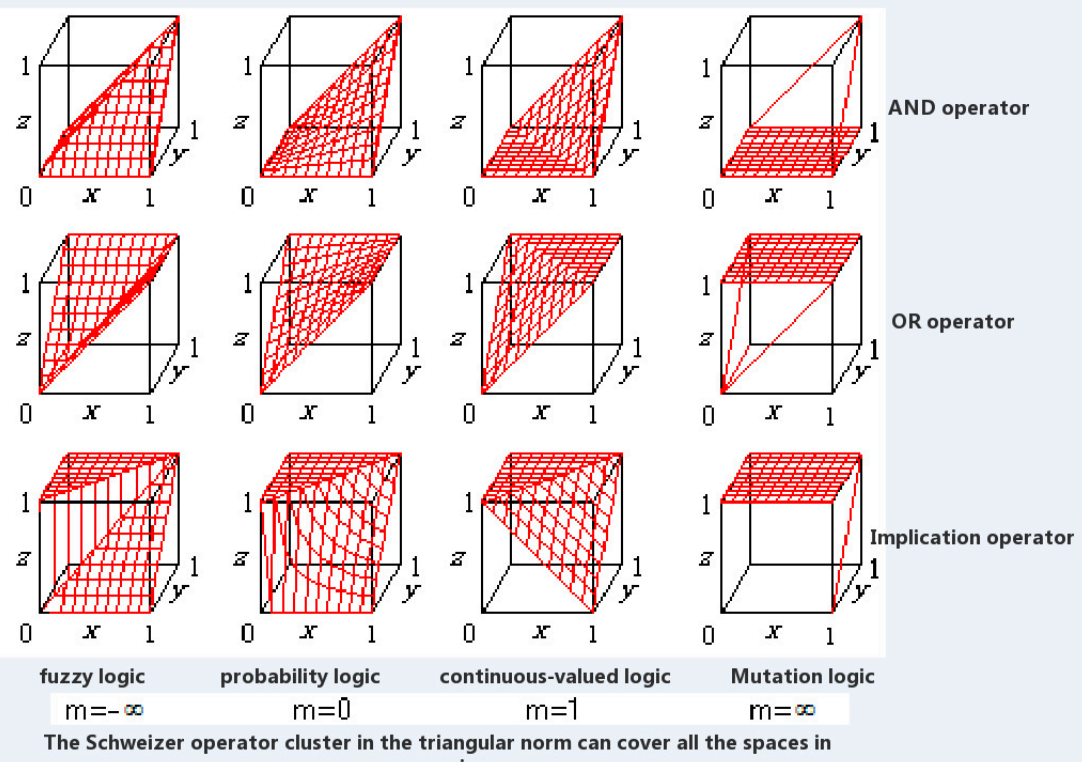

The Schweizer operator cluster in the triangular norm can cover all the spaces in succession

Figure 3. The four non-standard logic are four special points in the continuously changing Schweizer operator cluster.

\subsection{Find the Base Model of Flexible Propositional Logic}

In order to describe the flexible thinking ability of the human brain, Huacan HE put forward the concept of Universal Logics in 1996, published Universal Logics Principle in 2001, proposed Universal Logics research outline and established the flexible logic algebra and flexible propositional logic system. To commemorate the 50th anniversary of the birth of artificial intelligence, he published the English version of Universal Logics Principle in 2006.

The foundation result of establishing the flexible propositional logic is the findings of base model, which is the five operator groups of the Post continuous logic. The authors only increase the average operator and the combination operator, which can accommodate the uncertainty of the truth degree of proposition, $x, y, z \in[0,1]$. These base models specify the transformation relations between the two extremes 0,1 and the neutral transformation of the intermediate transition point $x \in(0,1)$ (Figure 4).

\section{NOT operator}

AND operator

OR operator

Implication operator

Equivalence operator

Average operator

Combination operator

$$
\begin{aligned}
& x=1-x \\
& x \wedge y=\Gamma[x+y-1] \\
& x \vee y=1-\Gamma[((1-x)+(1-y)-1)] \\
& x \rightarrow y=\Gamma[1-x+y] \\
& x \leftrightarrow y=1-|x-y| \\
& x^{\oplus} y=1-((1-x) / 2+(1-y) / 2)
\end{aligned}
$$$$
x(y)=\Gamma[x+y-\mathrm{e}] \text { where } \Gamma[] \text { is } 0,1 \text { limiting function. }
$$
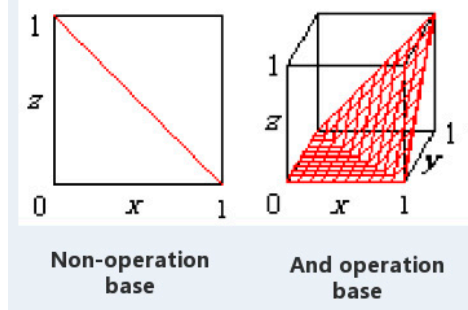

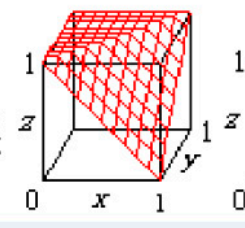

Implication operation base
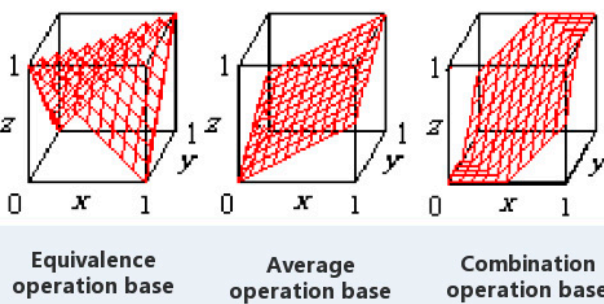

Figure 4. The base model of flexible propositional logic. 
Completeness Analysis of Base Model: First, the Boolean algebra is complete in binary information processing. It can describe all the output $\mathrm{z}$ of 16 different modes in binary information processing (transform). See Table 3 for details.

Table 3.The 16 modes of two-valued binary information processing.

\begin{tabular}{|c|c|c|c|c|c|c|c|c|c|c|c|c|c|c|c|c|c|}
\hline \multicolumn{2}{|c|}{ Input } & \multicolumn{16}{|c|}{ The Boolean Algebra Can Describe the Output z of All 16 Kinds of Information Processing (Transform) Modes } \\
\hline$x$ & $y$ & $\begin{array}{l}0 \\
\text { (1) }\end{array}$ & $\begin{array}{l}x \wedge \\
y(2)\end{array}$ & $\begin{array}{l}x \Lambda \\
y(3)\end{array}$ & $\begin{array}{l}x \\
\text { (4) }\end{array}$ & $\begin{array}{c}x \wedge \\
y(5)\end{array}$ & $\begin{array}{l}y \\
\text { (6) }\end{array}$ & $\begin{array}{c}x \neq y \\
(7)\end{array}$ & $\begin{array}{r}x \vee \\
y(8)\end{array}$ & $\begin{array}{l}x \wedge \\
y(9)\end{array}$ & $\begin{array}{c}x=y \\
\text { (10) }\end{array}$ & $\begin{array}{l}y \\
\text { (11) }\end{array}$ & $y \rightarrow x$ (12) & $\begin{array}{c}x \\
(13)\end{array}$ & $\begin{array}{c}x \rightarrow y \\
\text { (14) }\end{array}$ & $\begin{array}{c}x \vee \\
y(15)\end{array}$ & $\begin{array}{c}1 \\
16\end{array}$ \\
\hline 0 & 0 & 0 & 0 & 0 & 0 & 0 & 0 & 0 & 0 & 1 & 1 & 1 & 1 & 1 & 1 & 1 & 1 \\
\hline 0 & 1 & 0 & 0 & 0 & 0 & 1 & 1 & 1 & 1 & 0 & 0 & 0 & 0 & 1 & 1 & 1 & 1 \\
\hline 1 & 0 & 0 & 0 & 1 & 1 & 0 & 0 & 1 & 1 & 0 & 0 & 1 & 1 & 0 & 0 & 1 & 1 \\
\hline 1 & 1 & 0 & 1 & 0 & 1 & 0 & 1 & 0 & 1 & 0 & 1 & 0 & 1 & 0 & 1 & 0 & 1 \\
\hline
\end{tabular}

The two-valued binary neuron (perception) model (Figure 5) can be used to simulate the 16 types of information processing (transformation) modes by changing the internal state parameters a, b, e, as detailed in Table 4 .

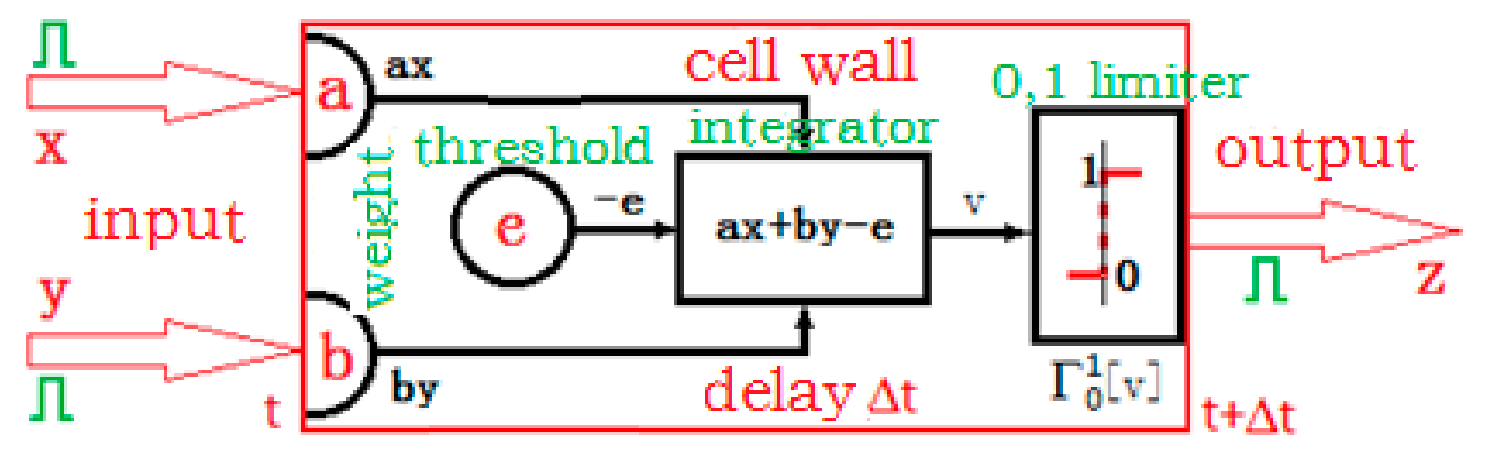

\section{The internal structure of two valued neurons}

Figure 5. Two-valued binary neuron (perception) model.

Table 4. The 16 state parameters in two-valued binary information processing.

\begin{tabular}{|c|c|c|c|c|c|c|c|c|c|c|c|c|c|c|c|c|}
\hline \multirow{2}{*}{ Parameter } & \multicolumn{16}{|c|}{ All 16 Types of Status Parameters of Information Processing (Transformation) Mode } \\
\hline & (1) & (2) & (3) & (4) & (5) & (6) & (7) & (8) & (9) & (10) & (11) & (12) & (13) & (14) & (15) & (16) \\
\hline $\mathrm{a}$ & 0 & 1 & 1 & 1 & -1 & 0 & & 1 & -1 & & 0 & 1 & -1 & -1 & -1 & 1 \\
\hline$b$ & 0 & 1 & -1 & 0 & 1 & 1 & implementation & 1 & -1 & implementation & -1 & -1 & 0 & 1 & -1 & 1 \\
\hline $\mathrm{e}$ & 0 & 1 & 0 & 0 & 0 & 0 & & 0 & -1 & & -1 & -1 & -1 & -1 & 2 & -1 \\
\hline
\end{tabular}

Secondly, the base model can inherit the completeness in the two-valued information processing at 0,1 endpoint. The new logical operation type brought by the presence of intermediate transition values, can be compensated by the average operator and the combination operator. It will be proved later that the adjustment function corresponding to various uncertainty parameters ensures that the processing of the intermediate transition value $x \in(0,1)$ is complete. (See Figure 6).
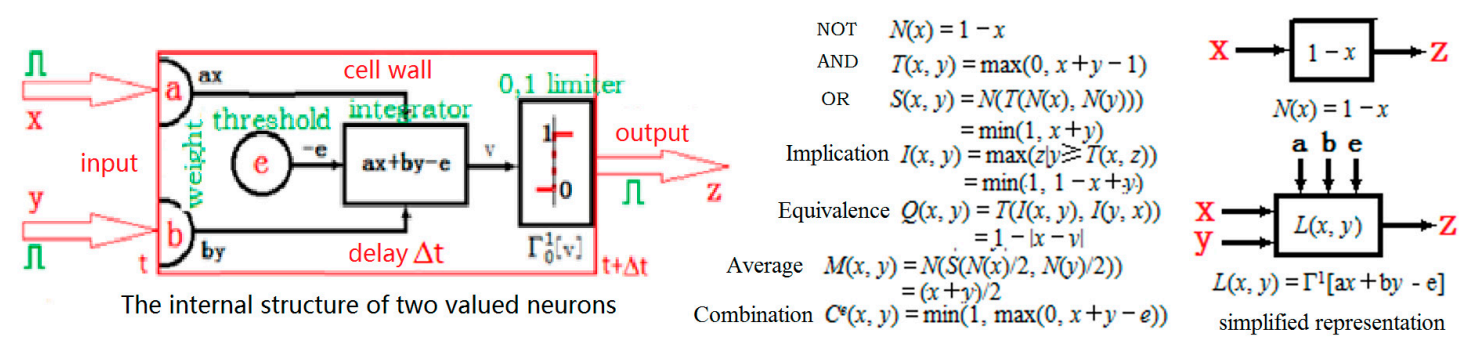


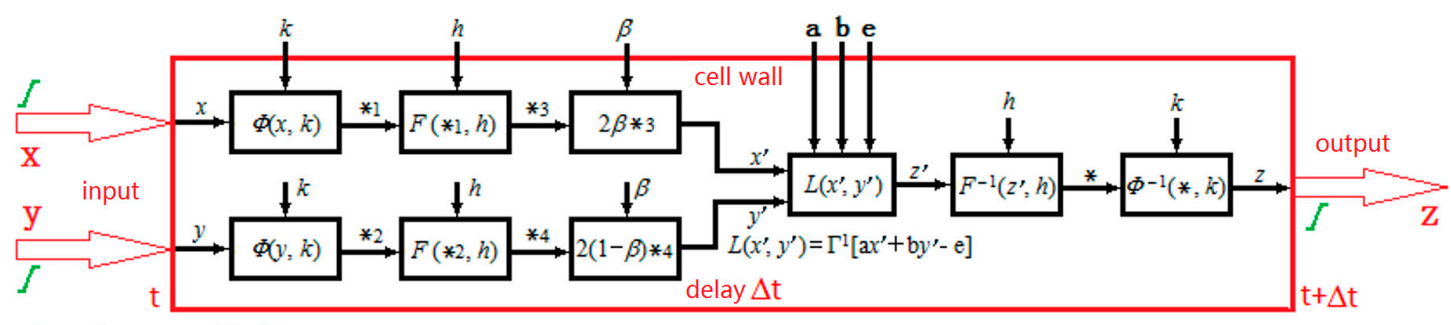

Internal structure of flexible neurons $\quad N(x, k)=\Phi^{-1}(N(\Phi(x, k)), k) \quad L(x, y, k, h, \beta)=\Phi^{-1}\left(F^{-1}(L(2 \beta F(\Phi(x, k), h), 2(1-\beta) F(\Phi(y, k), h), h), k)\right.$

where $F(x, h)=x^{m}, m \in(-\infty, \infty), m=(3-4 h) /(4 h(1-h)) \quad \Phi(x, k)=x^{n}, \quad n \in(0, \infty), n=-1 / \log _{2} k \quad L(x, y, \beta)=L(2 \beta x, 2(1-\beta) y)$

Figure 6. Complete continuous binary cell (perceptual) model.

\subsection{Find All the Uncertainties Accommodated in the Flexible Propositional Logic Operator}

The results show that there are five kinds of uncertainties in the flexible propositional logic operator, except for the uncertainty $x, y, z \in[0,1]$ of the flexible proposition itself. Also including the uncertainty of the proposition truth value error $k \in[0,1]$, the Uncertainty of Generalized Correlation between Two Propositions $h \in[0,1]$, the Uncertainty of Relative Weights between Two Propositions $\beta \in[0,1]$, the uncertainty of the true and false thresholds of the proposition $e \in[0,1]$. These uncertainties will affect the way and extent of the intermediate transition point $(0,1)$ deviating from the base model. (See Figure 7).

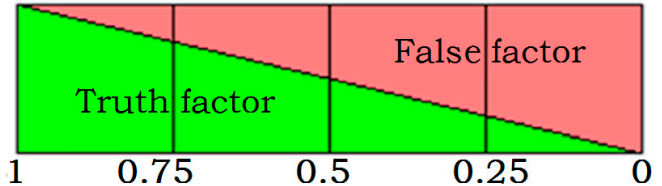

1. The uncertainty $x$ of truth value of proposition caused by contradiction between true and false

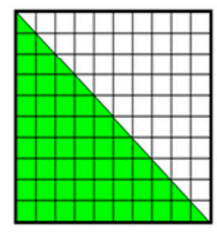

True -0.5 , False -0.5

2.The uncertainty $k$ contradiction between generousness and strictness

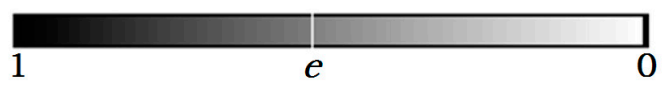

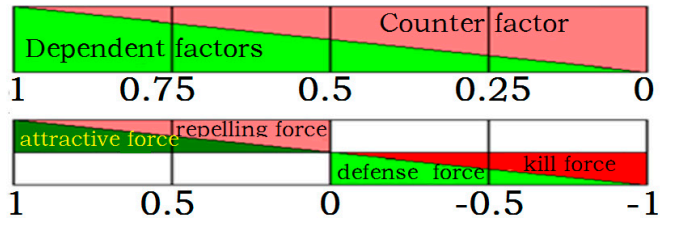

3.The uncertainty $h$ of the relationship between propositions caused by contradiction of friends and enemies

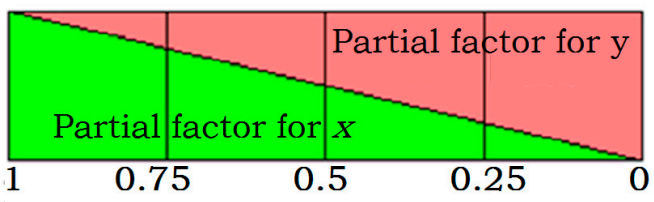

4. The uncertainty $\not \beta$ of propositional weights

caused by contradiction between likes and dislikes

5.The uncertainty $e$ of the true/false dividing line (threshold)

Figure 7. All the uncertainties that can be accommodated in the flexible propositional logic operator.

\subsection{Find the Way and Extent of Impact from Various Uncertainties on the Logical Operator Base Model}

The key to the success of Universal Logics research is to find the integrity cluster of tuning functions corresponding to uncertainty (Schweizer). It can correct the results of logical operations based on the base model, and ensure that such amendments have covered all possible spaces where this uncertainty should be affected. The mathematical tools we use are triangular norm theory, and rigorously proved on the basis of widely accepted NOT-axiom, AND-axiom, OR-axiom, contain-axiom, equivalence-axiom, average-axiom, and combine-axiom. (See Figure 8). 

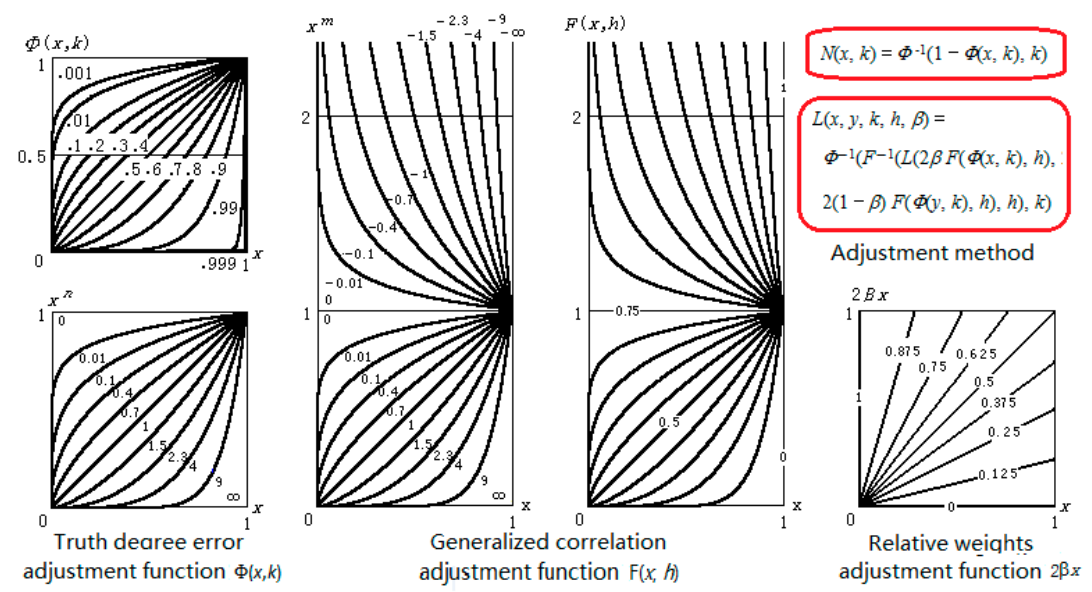

Figure 8. The way and extent of impact from various uncertainties on the logical operator base model.

\subsection{Integrity Cluster of Flexible Logic Operator}

0 -type flexible logic has only one base model. $L_{0}(x, y), x, y \in[0,1]$.

1-type flexible logic has three subtypes, which are all complete logical spectrum. $L_{k}(x, y, k), x, y$, $k \in[0,1], L_{h}(x, y, h), x, y, h \in[0,1], L_{\beta}(x, y, \beta), x, y, \beta \in[0,1]$

2-type flexible logic has three subtypes, which are all complete logical spectrum. $L k, h(x, y, k, h), x$, $y, k, h \in[0,1], L_{h \beta}(x, y, h, \beta), x, y, h, \beta \in[0,1], L_{k \beta}(x, y, k, \beta), x, y, k, \beta \in[0,1]$

3-type flexible logic has only one base model, which is complete logical spectrum. $L_{3}(x, y, k, h, \beta)$, $x, y, k, h, \beta \in[0,1]$

These complete logical spectra together form a perfect basic library of general propositional logic. And the basic operator needed for intelligent information processing is all in it. Other more complex information processing operators can be combined by these basic operators.

\subsubsection{Generalized Correlation on the Adjustment of Logic Operators}

With the adjustment function in the base mode, you can get a variety of binary logic operations of the complete operator cluster (See Figure 9).

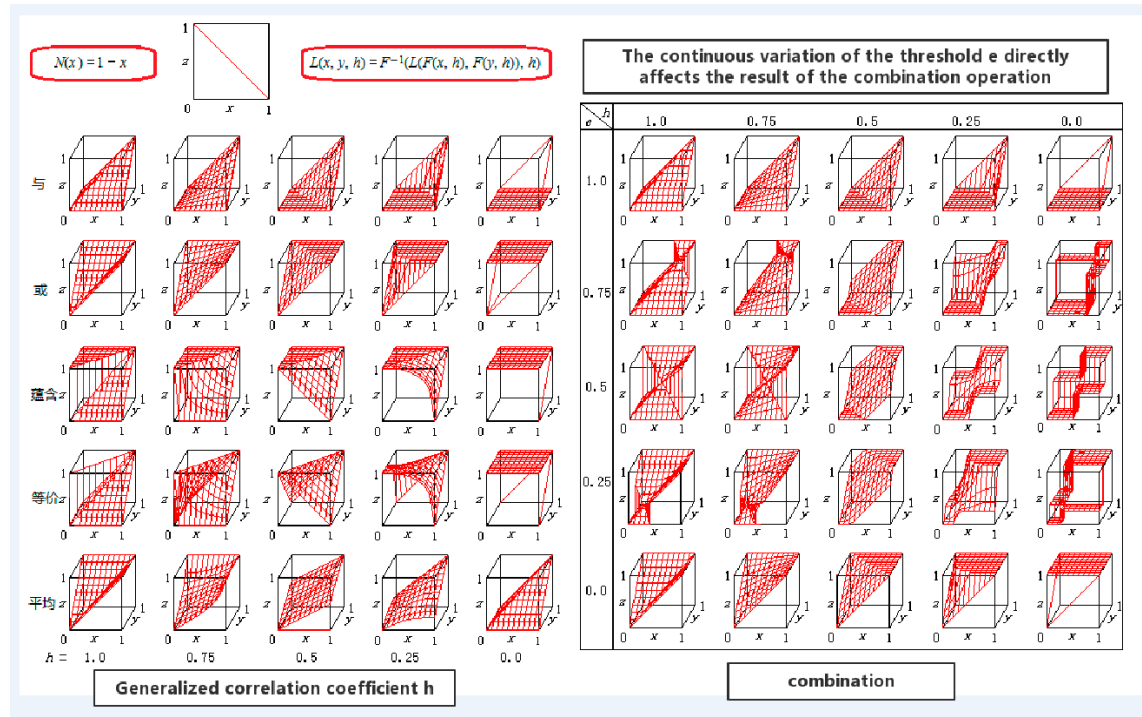

Figure 9. The impact of generalized correlativity on the results of the operation (use the sampling point to indicate the continuous trend). 
The binary operation $L(x, y, h)$ in Figure 9 is a one-dimensional propositional logic spectrum (see Figure 10). Only the impact of relevance is considered in the classical probability theory, including the maximum relative relationship $(h=1)$, the maximum repulsion $(h=0.5)$, and the middle of the dividing line is independent correlation $(h=0.75)$. They are compatible that can only be used to describe the difference between friends. This relation is constrained by the compatibility criterion and must satisfy the equation of $T(x, y, h)+S(x, y, h)=x+y$. In contrast, it further increase the phase relationship in the pan logic, which can be used to describe the degree of opposition between the enemies, including the maximum peace state $(h=0.5)$ and the maximum hostile state $(h$ $=0)$. The middle of the dividing line is stalemate $(h=0.25)$. In the phase relation, the $T(x, y, h)+S(x, y$, $h)=x+y$ criterion is satisfied except for the special point $h=0.25$. If there is a need for expansion of military preparations in peacetime, preparation criteria must meet the $T(x, y, h)+S(x, y, h)>x+y$. If there is a need to destroy the enemy during the war, killer guidelines must meet the $T(x, y, h)+S(x, y$, $h)<x+y$. In addition, three special points of AND, OR operator only are given in the classical probability theory where $h=1,0.75,0.5$. The other points can only be calculated by the conditional probability formula, and the Universal Logics gives the operator of all points. In this sense, the Universal Logics is a generalized probability theory considering generalized correlation. It is the first to combine the relevance of probability theory with the theory of self-existence, extended to generalized correlation $h$, and then combined with the triangular norm theory. The complete operator clusters of $h \in[0,1]$ are obtained by Schweitzer operator clusters, leading to the establishment of generalized probability theory. By introducing the influence of the measure error $k$ and the relative weight $b$, a complete operator library is formed which contains all the information processing operators, and the conditional probability. By introducing the influence of the measure error $\mathrm{k}$ and the relative weight $\mathrm{b}$ further, a complete operator library is formed which contains all the information processing operators and complements the conditional probability.

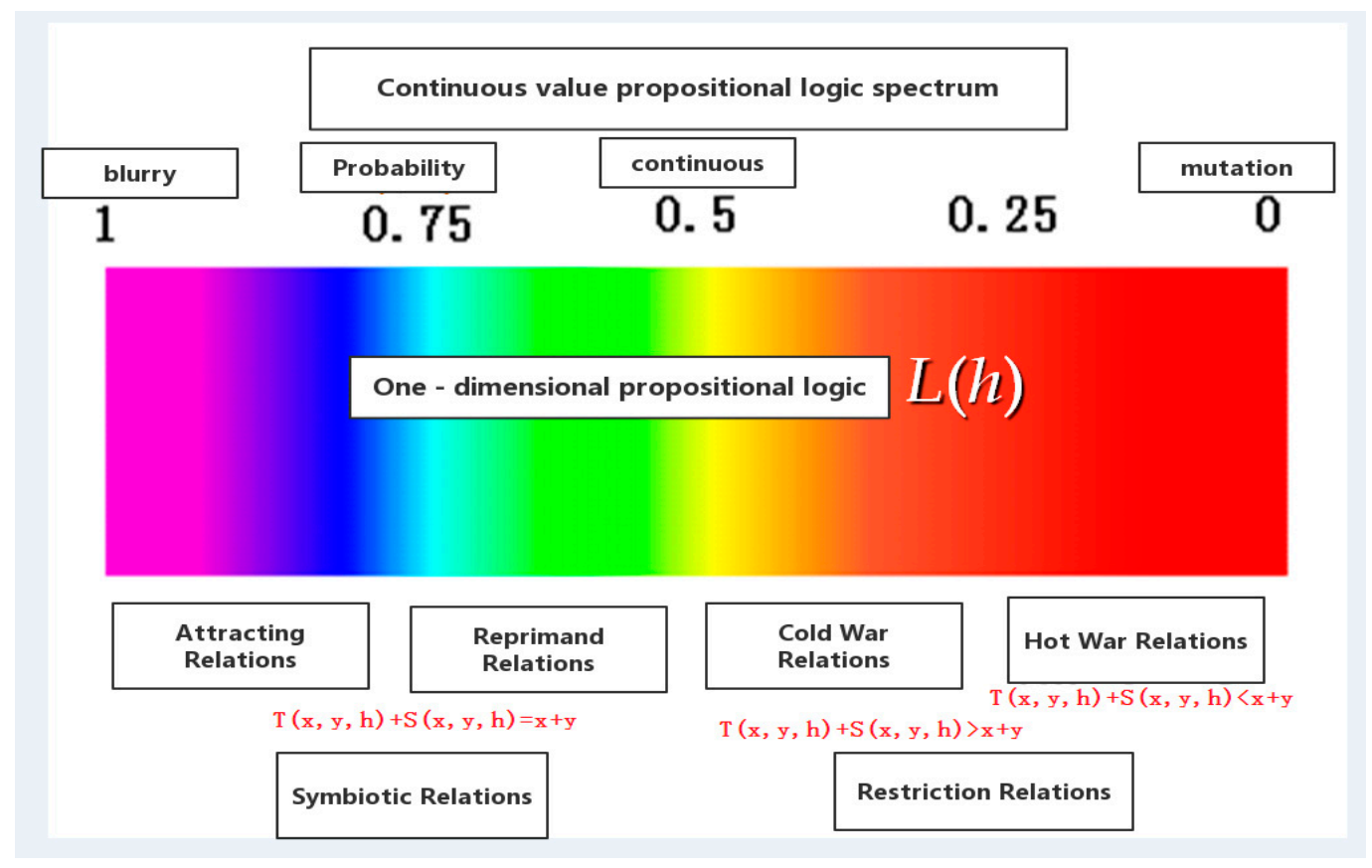

Figure 10.One-dimensional propositional logic spectrum $L(x, y, h)$.

\subsubsection{Generalized Correlation and Error Co-Ordination of Logical Operators}

It is possible to obtain a complete operator cluster for various logical operations If further introduction adjustment function $\Phi(x, k)=x^{n}$ for adjustment $\left(n=-1 / \log _{2} k, k \in[0,1]\right)$. The following NOR, AND, OR as an example to illustrate (See Figure 11). 


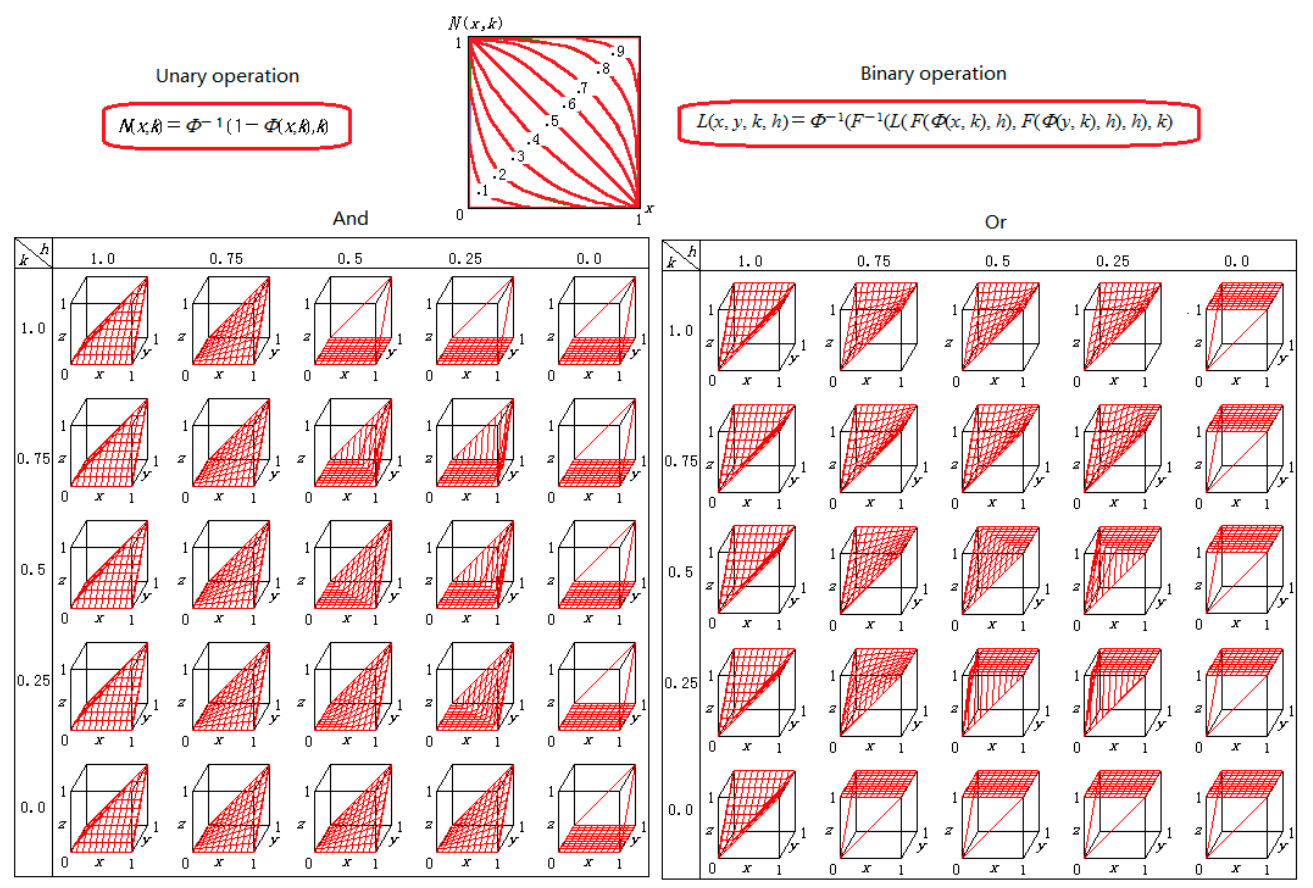

Figure 11. Influence of Generalized Correlation and Error on the Operation result (Use sampling point indicating continuous change trend).

From Figure 12, we can read that it can form the two-dimensional propositional logic spectrum $L(x, y, k, h)$ if the influence of the measurement error is further introduced in the generalized probability theory considering generalized correlation. And many uncertain reasoning theories exist in this way, they bring in the influence of measure error based on the classical probability theory, moreover, the possible measure, likelihood measure, trust measure and inevitable measure are put forward on the uncertain reasoning theories, which beyond the probability measure. In the pan-proposition logic, the two-dimensional propositional logic spectrum $L(x, y, k, h)$ not only contains these theoretical results, but also found a logical part of the phase., which is a new logic that has not been raised by our predecessors and it is useful for describing the widespread relationship between nature.

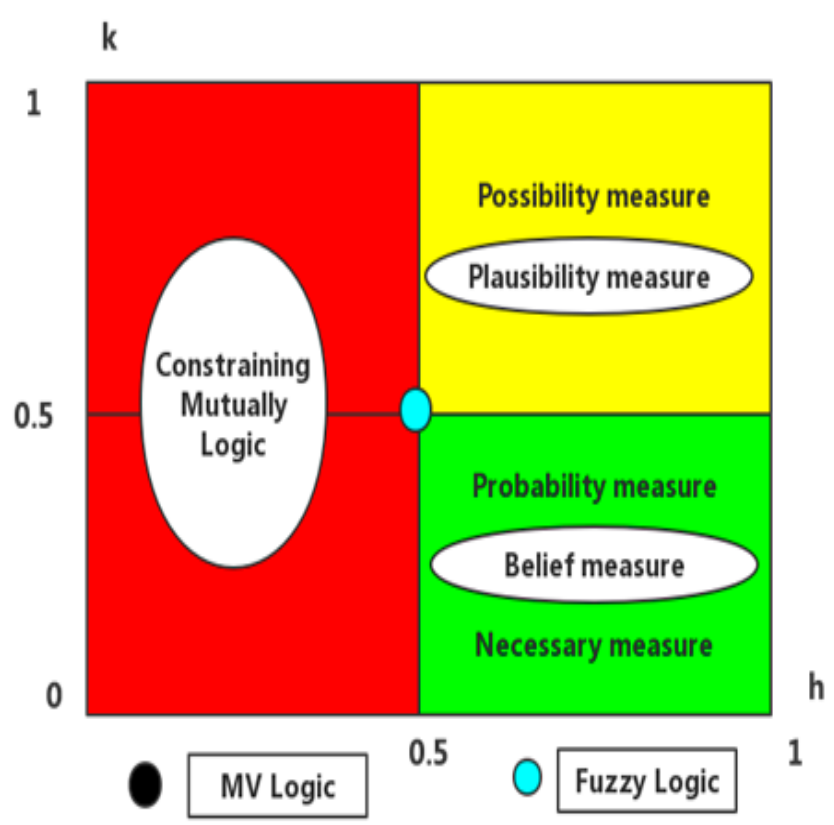

Figure 12. Two-dimensional propositional logic spectrum $L(x, y, k, h)$. 


\subsubsection{Generalized Correlation and Relative Weights for the Adjustment of Logical Operators}

Let the weight assignment be $x: \beta ; y: 1-\beta, \beta=0.5$ is the equal weight operation. Take the average operation for example (See Figure 13):

Equal rights average: $M(x, y, h, 0.5)=M(x, y, h)=1-\left(\left((1-x)^{\left.\left.m+(1-y)^{m}\right) / 2\right)^{1 / m}}\right.\right.$.

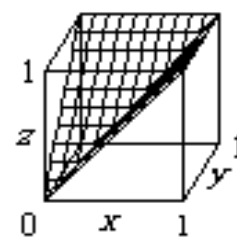

$h=1.0$

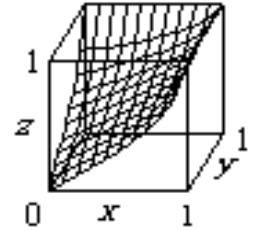

$h=0.75$

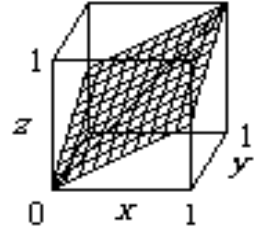

$h=0.5$

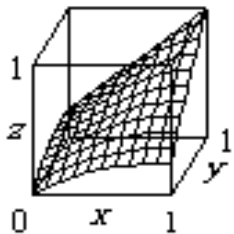

$h=0.25$

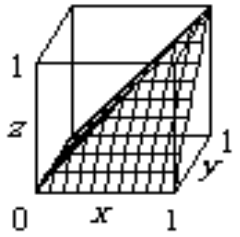

$h=0.0$

Weighted average: $M(x, y, h, 0.6)=1-\left(0.6(1-x)^{m}+0.4(1-y)^{m}\right)^{1 / m}$.
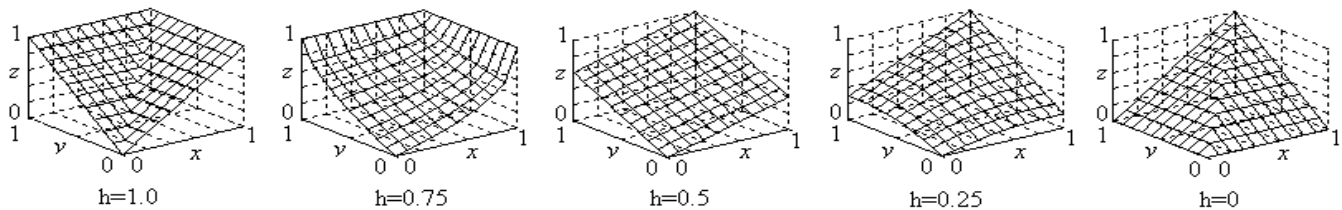

Figure 13. Equivalence of weighted average and weighted average. Sampling point for indicating the continuous change trend.

\subsection{Discovery the Integrity of the Logic}

(1) The soundness of standard logic has been contained in reliability and completeness, and does not require special consideration.

(2) After introducing all kinds of uncertainties into the Universal Logics, the reasoning result becomes indefinite. The original logical operate of isolated existence is expanded into a continuous change logical operator spectrum, which contains an infinite number of operators changes in uncertain parameters.

(3) The logic operator spectrum with h parameter is the most basic logic operator spectrum. The flexible logic operator spectrum with $k, h, \beta$ parameters lays the mathematical foundation for establishing the complete propositional logic.

(4) Continuous value logical reasoning is the process of coexistence of symbolic calculus and numerical calculation. The result is still a continuous value. There must be an intermediate transition value $x \in(0,1)$ the two extremes 0 and 1 . The reliability and completeness of the standard logic can only guarantee that the propositional truths do not distort at $x \in\{0,1\}$ in the process of deduction, there is no guarantee that the intermediate transition value $x \in\{0,1\}$ does not cause distortion. The anomalous results that appear in some nonstandard logic are a manifestation of this distortion. In order to avoid the possibility of distortion of the intermediate transition value $x \in(0,1)$ in the process of deduction, the sufficient and necessary conditions for the flexibility of the flexible logic are proposed as follows:

(5) Definition: a sound flexible logic system must have the following basic properties:

L1:Idempotent law $P \wedge P=P$

L2:Idempotent law $P \vee P=P$

L3: Contradiction law $\sim P \wedge P=0$

L4:Law of excluded middle $\sim P \vee P=1$

L5:Involution law $\sim P=P$

L6:MP rules $P, P \rightarrow Q \models Q$

(6) It canbe proved h-type flexible logic spectrum is a sound logic system, it satisfies:

L1:Idempotent $\operatorname{law} T(x, x, 1)=x$ 
L2:Idempotent law $S(x, x, 1)=x$

L3:Contradiction $\operatorname{law} T(N(x), x, 0.5)=0$

L4:Law of excluded middle $S(N(x), x, 0.5)=1$

L5:Involution law $N(N(x))=x$

L6:MP rulesT $(x, I(x, y, h), h) \leq y$

$k h$-type flexible spectrum is a sound logic system, it is satisfied that:

L1:Idempotent $\operatorname{law} T(x, x, 1)=x$

L2:Idempotent $\operatorname{law} S(x, x, 1)=x$

L3:Contradiction $\operatorname{law} T(N(x), x, 0.5)=0$

L4:Law of excluded middle $S(N(x), x, 0.5)=1$

L5:Involution law $N(N(x))=x$

L6:MP rules $T(x, I(x, y, h), h) \leq y$

The basic principle of ensuring logical integrity is: (1)The truth of two propositions may be the same, but $\mathrm{h}$ is not necessarily 1 , and the truth of the same proposition must be the same, and $h$ must be 1. (2)As long as $k$ the same, soundness must be guaranteed.

\section{The Application on Reasoning Calculation and Online Identification}

Universal logic, as a kind of framework, it can be used in many cases [4-8]. There are two applications of them.

\subsection{Discovering the Complete Clusters in Propositional Universal Logics}

It is a significant event that discovering the complete clusters in propositional universal logics. Such clusters contain all possible propositional operators in intelligent information processing. Each of them is a complete propositional operator of intelligent information processing. Although it has a weakness that the computing process is too complex to be accepted, we can get rid of this by saving the clusters in disks instead of computing it as soon as we need it. It is acceptable because such clusters has absolute integrity. By doing so, every clusters are on tap and intelligent information processing can get result directly, avoiding undesired computing trouble. Within intelligent information processing, there are two ways to use operator clusters:

(1) As to logical reasoning, we can use such clusters directly. By calculating operator-type parameters $(\mathrm{a}, \mathrm{b}, \mathrm{e})$ and uncertainty parameters $(k, h, \beta)$, we can chose the appropriate operator and get the result.

(2) As to machine learning and data mining, we can use such clusters indirectly. Through on-line data processing we can distinguish operator-type parameters $(a, b, e)$ and uncertainty parameters $(k, h, \beta)$ of each logics node( like neuron). Automatically, we can identify logic behavior of processing objects.

\subsection{Application in Many-Dimensions Flexible Logics and Many-Valued Logic}

Such flexible logics, which can be represented as $[0,1]^{n}$, is a transformation of one-dimension continuous logic:[0,1], by using dimension expansion rules. On the other hand, we can use depreciation rules to generate finite many-valued logics, such as three-valued logic: $\{0, u, 1\}$ and five-valued logic: $\{0, \mathrm{pf}, \mathrm{u}, \mathrm{pt}, 1\}$ (see Figure 14). 


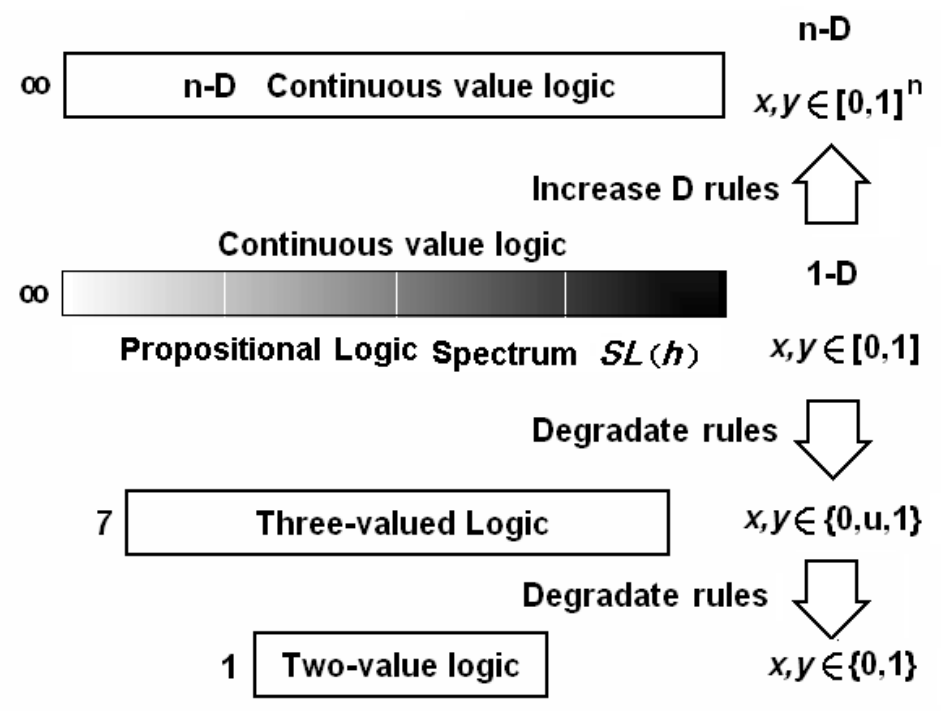

Figure 14.Extending applications by dimension expansion rules and depreciation rule.

\section{Future Work}

The next work will be building one-dimension universal logic predicate and adding different flexible logics quantifier. Flexible quantifiers defined on $\mathrm{W}$ includes: threshold quantifier representing propositional deviation: $0^{k}$; hypothetical quantifier representing trust degree of hypothetical proposition: $\$$; range quantifier restricting change of individuality: $\oint^{k}$; positional quantifier indicating the relative place of individuality: $\$ k$; transition quantifier changing the distribution of value: $f^{k}$, etc. Additionally, $k \in[0,1]$ is a constraint condition, which is also called degree flexibility, to describe the uncertainty of constraint.

It is a long process, for there remains many uncertainty in mathematical treatment. Universal Logics will develop progressively into a perfect dialectic logic.

Conflicts of Interest: The authors declare no conflict of interest.

\section{References}

1. He, H.; Liu, Y.; He, D. Generelized logic in experience thinking. Sci. China (Ser. E) 1996, 39, 225-234.

2. He, H.; Wang, H.; Liu, Y.; Du, Y. Principle of Universal Logics; Science Press: Beijing, China, 2001.

3. He, H.; Wang, H.; Liu, Y.; Du, Y. Principle of Universal Logics; Science Press: Beijing, China; NWPU Press: Xi'an, China, 2006.

4. He, H.; Ai, L.; Wang, H. Uncertainties and the Flexible Logics. In Proceedings of the IEEE 2003 International Conference on Machine Learning and Cybernetics, X'an, China, 2-5 November2003; Volume 4/5, pp. 2573-2578.

5. Zhou, Y. Research and Application of Association Knowledge Mining Algorithm. Ph.D. Thesis, Northwestern Polytechnical University, Xi'an, China, 2000.

6. Chen, Z. Studies on Correlation Reasoning of Fractal, Chaos, and Logic in Complex System. Ph.D. Thesis, Northwestern Polytechnical University, Xi'an, China, 2004.

7. Mao, M. Studies on Operation Model and Reasoning of Object-OrentiedGaneraliedSaptial Logic. Ph.D. Thesis, Northwestern Polytechnical University, Xi'an, China, 2006.

8. Fu, L.; He, H. Studies on control method based on llexible logic. Comput. Sci.2009, 36, 158-161. 